\title{
A study on the impact of the LHAASO-WCDA photomultiplier tube afterpulse
}

\author{
Baomin Chen \\ Institute of High Energy Physics, CAS \\ E-mail: chenbmeihep.ac.cn
}

\section{Bo Gao}

Institute of High Energy Physics, CAS

E-mail: gaobolihep.ac.cn

\section{Min Zha}

Institute of High Energy Physics, CAS

\section{Zhiguo Yao}

Institute of High Energy Physics, CAS

\section{Mingjun Chen}

Institute of High Energy Physics, CAS

\section{Zhaoguang Pang}

Hebei Normal University

\begin{abstract}
The Water Cerenkov Detector Array (WCDA) is an important part of Large High Altitude Air Shower Observatory (LHAASO). It is mainly composed of large photomultiplier tubes (PMT), including 8-inch and 20-inch in diameter. In the PMT test, the afterpulse signal time distribution shows a specific structure in time of $20 \mu$ s range after the main pulse. The afterpulse ratio also varies under different main pulse intensity, which will have an effect on the shower trigger and data taking. In this paper, some characteristics of the afterpulse and the influence of light intensity on the afterpulse are studied. It can help us understand performance of photomultiplier tubes and improve the data analysis of WCDA.
\end{abstract}




\section{Introduction}

The Large High Altitude Air Shower Observatory (LHAASO) is a major scientific infrastructure planning project for cosmic ray and high energy gamma astronomical experimental research. LHAASO-WCDA is one of the important components. The main physical target of the experiment is the full-sky detection of very high-energy gamma astronomy, including the detection and monitoring of very high-energy gamma sources, the measurement of energy spectrum and the study of extended morphology. The photomultiplier tube is the main component of the detector. When the photomultiplier tube is operated with a pulse signal, some small amplitude pulses can be seen after the main pulse. Such signals are called afterpulses.

The afterpulse of PMT used in WCDA has been investigated in USTC. In the test, the main pulse is set to $30 \mathrm{PE}$, using the by using commercial electronics (FADC and multi-hit TDC), the pulses which is larger than $1 / 3 \mathrm{PE}$ from $100 \mathrm{~ns}$ to $16 \mu$ s after the main pulse are counted, the afterpulse timing structure is shown in Fig.1[1], in this figure the main pulse arriving time is set to $t=0$. There are two kinds of afterpulses, one comes from nanoseconds after the main pulse which are the electron s reflected by the first dynode increases to the signal near the photocathode. The other comes from microseconds after the main pulse, mainly due to the formation of ion feedback by residual gas. The afterpulse rate is defined as the probability of averaging the afterpulse each individual photoelectron is generated in the main pulse, it is calculated as following function, the $\boldsymbol{N}_{\text {afterpulse }}$ is the sum of afterpulses counted in all time window (100 ns - $20 \mu$ s), the $\Sigma$ npe is the sum of main pulse charge in all triggers.

$$
\text { Afterpulse Rate }=\boldsymbol{N}_{\text {afterpulse }} / \text { Inpe }
$$

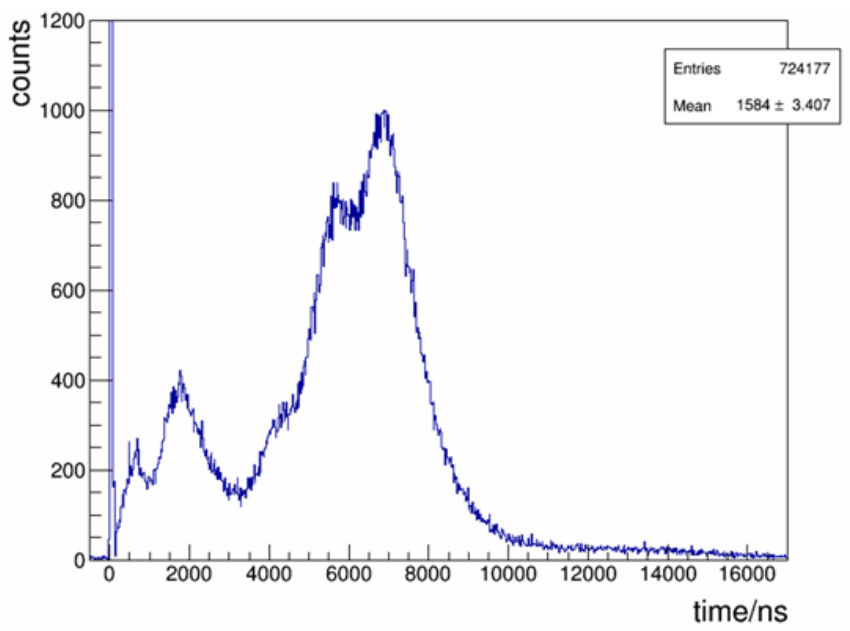

Figure 1: Time structure of pulse distribution

\section{Afterpulse test}

In WCDA, the charge given by PMT can be from 1 PE to more than 4000 PE in EAS measurement, for large cosmic ray shower (eg. 100TeV), the PMTs in shower core area can be saturated, and the probability of afterpulse is $100 \%$, which may cause fake "air showers" and trigger the whole array. To known the probability these fake showers, the afterpulse rate under different pulse light intensity need to be studied. 
On the other hand, the FEEs [2] for WCDA 1st pool have been manufactured and have been tested, so we use the FEE to test the PMT afterpulse rate. To simulate the outtrigger test mode, a two channel function generator is used, one channel fed a standard NIM pulse into one FEE channel for reference trigger signal, the other channel gave a positive pulse to trigger a LED which illuminated the PMT. The frequency is set to $1 \mathrm{kHz}$ to ensure the PMT can handle. The LED trigger pulse is set to different voltage to change the pulse light intensity. Test schematic diagram shown in Figure 2

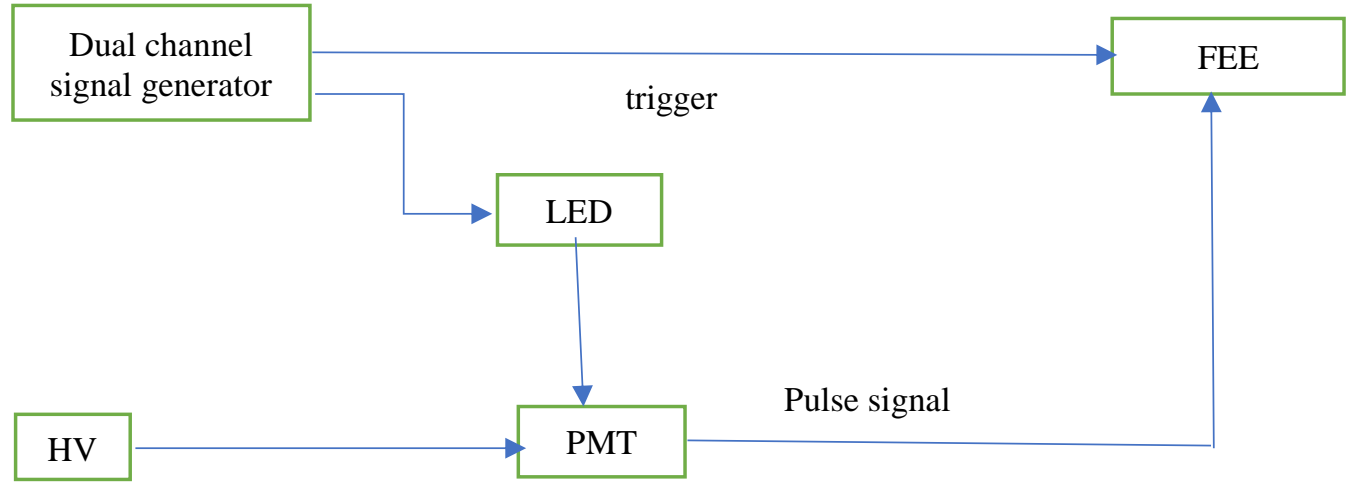

Figure 2: FEE's afterpulse test chart

\section{1 The relationship between 8-inch PMT light intensity and afterpulse}

The afterpulse that occurs over a period of time after the initial photoelectron signal is one of the background noise. In order to reduce the influence of afterpulse on the signal, the relationship between light intensity and afterpulse is studied in this paper.

WCDA's Pool 1 currently uses an 8-inch large PMT that illuminates an 8-inch photomultiplier tube using the light intensity of the LED. Figure 3 shows the waveform of the afterpulse signal at different light intensities in the range of 0-800 pe.. The light intensity in the figure from low to high is 45 pe, 148 pe, 308 pe, 452 pe, 637 pe, 667 pe, and the time interval is in the main 100-20000 ns after the pulse. By comparing the different light intensity afterpulse signals, it can be seen that the afterpulse entries have changed and the afterpulse rate has also changed. The relationship between light intensity and afterpulse rate is shown in Figure 4. It can be seen that the afterpulse rate of the 8-inch PMT decreases as the npe increases..

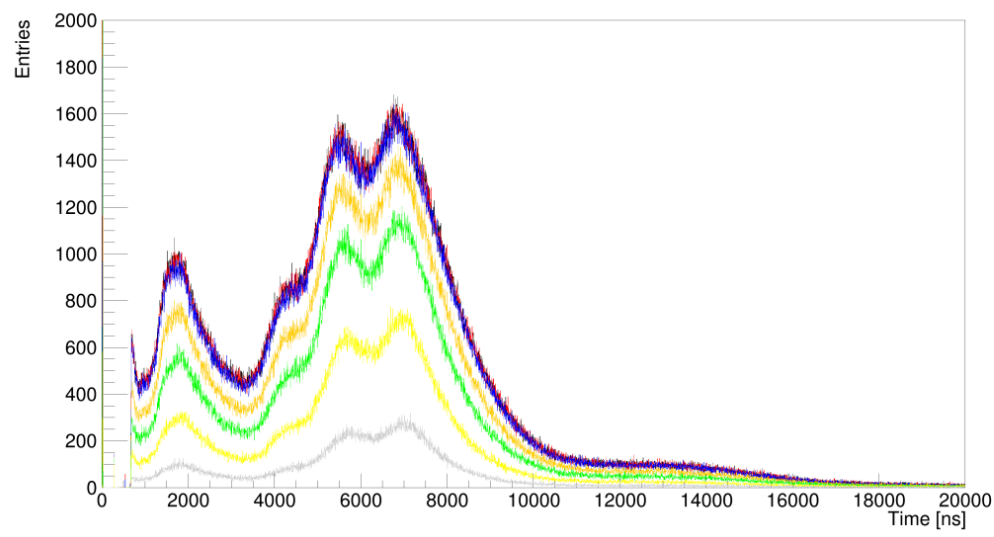

Figure 3: Afterpulse signal at different light intensities 


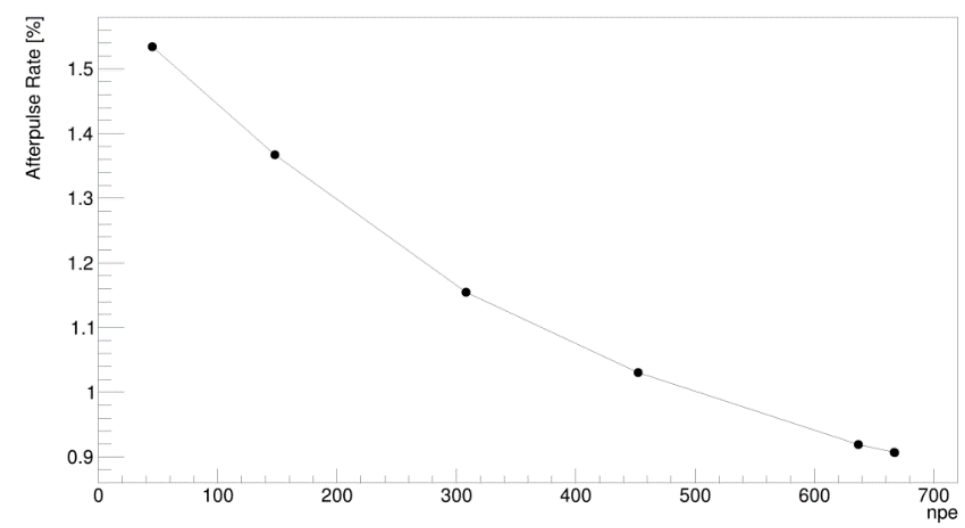

Figure 4: Relationship between light intensity and afterpulse rate

\subsection{The relationship between 20-inch PMT light intensity and afterpulse}

To detect cosmic ray signals in different energy segments, WCDA's 2nd and 3rd pools use a 20-inch PMT. Therefore, in order to reduce the background noise signal collected by the detector, it is necessary to study the afterpulse of different size PMT.

After the 20-inch PMT pulse test, the signal source gives three amplitude signals with a frequency of $1 \mathrm{kHZ}$ and a width of $15 \mathrm{~ns}$. The corresponding signal sizes are $50 \mathrm{pe}, 200 \mathrm{pe}$, and 500 pe, respectively. A 20-inch PMT is measured. The relationship between light intensity and afterpulse in the 20 microsecond time window is shown in Figure 5. Comparing Figures 4 and 5, it can be seen that the pulse rate after the 20-inch PMT decreases as the npe increases, which is the same as the 8-inch PMT.

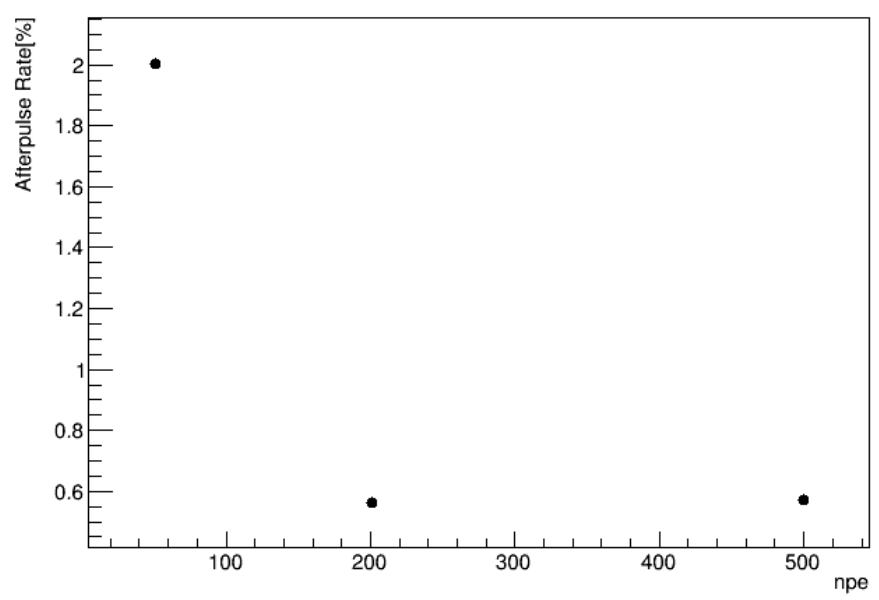

Figure 5: Relationship between light intensity and afterpulse rate

\section{Conclusion}

In this paper, the afterpulse signal of the PMT after the main pulse of the pulse signal is studied. The afterpulse rate is the main performance parameter for detecting the background noise signal of the detector. In the 800pe range.The afterpulse rate of different sizes of PMT is different, but the afterpulse rate decreases with the increase of light intensity, so the light intensity is controlled in a small range and the afterpulse has less influence on the detector. It is of great significance for the calibration work and data analysis of the detector. 


\section{Acknowledgments}

This work is supported by the National Key R\&D programe of China (No.2018YFA0404201) and the Chinese Academy of Sciences, the Key Laboratory of Particle Astrophysics, CAS

\section{References}

[1] X. Zhao. Afterpulse measurement for 8-inch candidate PMTs for LHAASO.

[2] Lei Zhao. Prototype of the Readout Electronics for WCDA in LHAASO.

[3] V.A.Morozov .Investigation of ion-feedback afterpulse spectra by the autocorrelation method.

[4] Q. An .Performance of a prototype water Cherenkov detector for LHAASO project.

[5] K. J. Time and Amplitude of Afterpulse Measured with a Large Size Photomultiplier Tube. 